\title{
Deterministic and random synthesis of discrete chaos
}

\author{
Miguel Romera $^{1 *}$, Michael Small ${ }^{2}$, Marius-F. Danca ${ }^{3}$ \\ ${ }^{1}$ Instituto de Física Aplicada, Consejo Superior de Investigaciones Científicas, Serrano 144, 28006 Madrid, Spain \\ ${ }^{2}$ Electronic and Information Engineering, Hong Kong Polytechnic University \\ ${ }^{3}$ Department of Mathematics, "Tehnofrig" Technical College, 3400 Cluj-Napoca, Romania
}

\begin{abstract}
In this paper two anticontrol algorithms for synthesis of discrete chaos are introduced. In these algorithms, the control parameter of a discrete dynamical system is switched, either randomly or in a deterministic way, between two or more values corresponding to periodic motions, the result being chaotic behavior. These algorithms require no knowledge of specific mathematical properties of the underlying map modeling the system. The existence of chaos is demonstrated using various tools including graphical iteration, histogram, Lyapunov exponent and surrogate tests. In this paper, these very simple and implementable chaotifiers are applied to the logistic map.
\end{abstract}

Keywords: Logistic map; Discrete chaos; Anticontrol; Superstable orbits; Surrogate tests.

\section{Introduction}

The chaotification of a dynamical system (variously also described as chaos synthesis, chaos generation, or anticontrol of chaos) represents a significant nontrivial problem and has seen a very rapid development (see for example [1] and [2] for discrete dynamical systems, [3] and [4] for continuous dynamical systems, and [5] for discontinuous dynamical systems).

Chaos synthesis seems to be a continuously expanding area, due to the great potential of chaos in non-traditional applications. The presence of chaos has been observed to be beneficial and even desirable in many domains, including mechanical, electronic, optical, and biological systems. In particular, the clinical study of human heart-beat analysis and regulation is one specific example of great relevance ([6] and references therein, [7]).

The most widely known method to make an arbitrarily given system chaotic or to enhance the existing chaos of a chaotic deterministic system is the use of small controls via time-delay feedback. Briefly, anticontrol in the case of a general one-dimensional discrete dynamical system $x_{n+1}=f\left(x_{n}\right)$, $x_{0} \in M \subseteq \mathbb{R}, f: M \rightarrow \mathbb{R}$, originally neither chaotic or complex nor ill-behaved or unstable, consists of finding a control sequence $\left\{u_{n}\right\}$ such that the output of the controlled system $x_{n+1}=f\left(x_{n}\right)+u_{n}$ is chaotic.

In this paper we introduce two anticontrol algorithms which switch the control parameter of a discrete dynamical system in (respectively) a deterministic or random ${ }^{1}$ manner between two or more

\footnotetext{
* Corresponding author.

E-mail addresses: miguel@iec.csic.es (Miguel Romera)

${ }^{1}$ In this paper by "random" (stochastic) number one understands a real number generated uniformly in the interval $[0,1]$, even it is actually a "pseudo" random due to the limits of computation.
} 
values of the control parameter for which the system exhibits stable dynamics. By doing so, we show that the originally non-chaotic system exhibits chaotic dynamics.

Within the framework discussed above for discrete chaos synthesis we have observed the following three possibilities (Table 1)

Table 1

Possibilities for discrete chaos synthesis

order + order $=$ chaos

order + chaos $=$ chaos

chaos + chaos $=$ chaos

In this paper we focus on the first case which is certainly the most surprising, and also seems to be the most interesting.

This kind of synthesis can be thought as representing a realistic model for many natural processes and resembles Parrondo's philosophy in a winning game: "losing + losing = winning" ([8] and [9]; in [10] a different Parrondian paradoxical phenomenon: "chaos + chaos $=$ order" is analyzed). In our paper the anticontrol algorithm could be read as "order + order = chaos".

In order to prove the chaotic character of behavior of the system obtained by the application of these two mentioned algorithms, we will use tools such as: time series, graphical iteration, histogram and Lyapunov exponent. Moreover we apply standard surrogate tests [11] and more recent pseudoperiodic surrogate tests [12]. Briefly, the purpose of these surrogate tests (the details are described in the Appendix) is to provide a statistical basis for classify the observed time series as stochastic, periodic or chaotic. In each case, artificial (surrogate) data consistent with the hypothesis of interest (for example linear noise or a linear periodic orbit) is generated. Statistical measures of this data are then taken and compared to the same quantities estimated for the original data (correlation dimension, Lyapunov exponents and algorithmic complexity are all popular choices). If the data and surrogates differ (in a statistical sense) then one can say that the hypothesis is rejected. If no difference is observed then no such conclusion can be made. The details of these algorithms have been described at length elsewhere (for example [13]) and are not the main focus of the current work. For completeness, we describe the necessary mechanics in the Appendix.

Our anticontrol algorithms could explain why when two or more stable discrete dynamical systems (e.g. populations, networks etc.) "meet", the result could be, generally, chaotic despite the fact that the underlying systems have ordered deterministic dynamics. Alternative, standard interpretation is that for two competing populations (with continuous mathematical model) we have a 2-D continuous system: chaos is not possible. Add more species, and chaos becomes possible, add more and more and it becomes simply statistically more likely.

Also one can imagine practical implementations especially in biology or medicine: life is only possible when there is no equilibrium; equilibrium means death, or a dynamical system without equilibrium means stochastic or chaotic system.

It is widely accepted (although not rigorously stated), that a mathematical classification of dynamical systems typically places chaotic dynamical systems near stochastic systems. The difference between these two classes being the level of stochasticity or the dimension of the nonlinear dynamics utilized (see e.g. [14] or [15]). The nature of our obtained systems places them somewhere between these two classes. However, we assert that the observed dynamics are closer to chaos than stochasticity.

The anticontrol was applied to several examples, but in this work we present only the results for the logistic map, that is well known and particularly simple.

The paper is organized as follows. In Section 2 the random anticontrol algorithm is presented and applied to the case of logistic map, while in Section 3 the deterministic anticontrol scheme is analyzed and applied to the same logistic map. Section 4 presents some concluding remarks. Finally, in the Appendix we present the surrogate techniques used to provide evidence of chaos in the cases of random and deterministic anticontrol algorithms. 


\section{Random anticontrol algorithm}

Let us consider a discrete deterministic dynamical system $S$ described by the following mathematical model $x_{n+1}=f_{p}\left(x_{n}\right), n=0,1,2, \ldots$ where $f_{p}: M \rightarrow \mathbb{R}$ is a real parametrized map, $x_{0} \in M$, and $p \in \mathbb{R}$ is the control parameter. Let $\mathcal{P}=\left\{p_{1}, p_{2}, \ldots, p_{N}\right\}$ be the set of $N \geq 2$ parameter values for which $S$ behaves stable.

By switching the $p$ value randomly at every step $n$ between the values of the set $\mathcal{P}$ one obtains a new system $S^{*}: z_{n+1}=f_{p}\left(z_{n}\right), f_{p}: M \rightarrow \mathbb{R}, z_{0}=x_{0} \in M, n=0,1,2, \ldots$

Let us denote by $p \triangleright \mathcal{P}$ the random choice of $p$ from among the first $r \leq N$ values of the set $\mathcal{P}$. That is, at every step $n, p=p_{i} \in P$, for $i=\operatorname{rand}(r)$, rand being one of the many standard pseudorandom number generators.

Thus, the random anticontrol algorithm (RA) generates the sequence

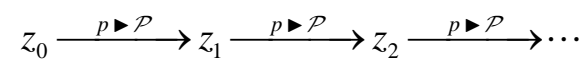

The corresponding algorithm is described by the following pseudocode

$$
\begin{array}{|l|}
\hline \text { Choose } z_{0} \\
\text { For } n=0 \text { to } n_{\max } \\
p \triangleright \mathcal{P} \\
z_{n+1}=f_{p}\left(z_{n}\right) \\
\text { next } \\
\hline \text { RA algorithm }
\end{array}
$$

For the sake of simplicity of presentation, we fix $r=2$. That is, $p$ is switched randomly between only two certain values of $\mathcal{P}$ which will be denoted $\left(p_{1}, p_{2}\right)$. We will name $S_{1}$ and $S_{2}$ the system $S$ with, respectively, $p_{1}$ and $p_{2}$ parameter values and $S^{*}$ the obtained system.

As an example, let us consider the logistic map. As is very well known, the logistic map has the following mathematical form: $f_{p}=p x(1-x) p \in[0,4]$ with $M=[0,1], f:[0,1] \rightarrow[0,1]$.

In order to obtain the most rigorous possible results we will use for $\mathcal{P}$ the values corresponding to superstable orbits of period 3, 4, .., 7 presented in Table 2. 
Table 2

The $\mathcal{P}$ set containing the parameter values corresponding to the superstable period $3,4, \ldots, 7$ orbits of the logistic map in the chaotic zone $p \in[3.56994567,4]$.

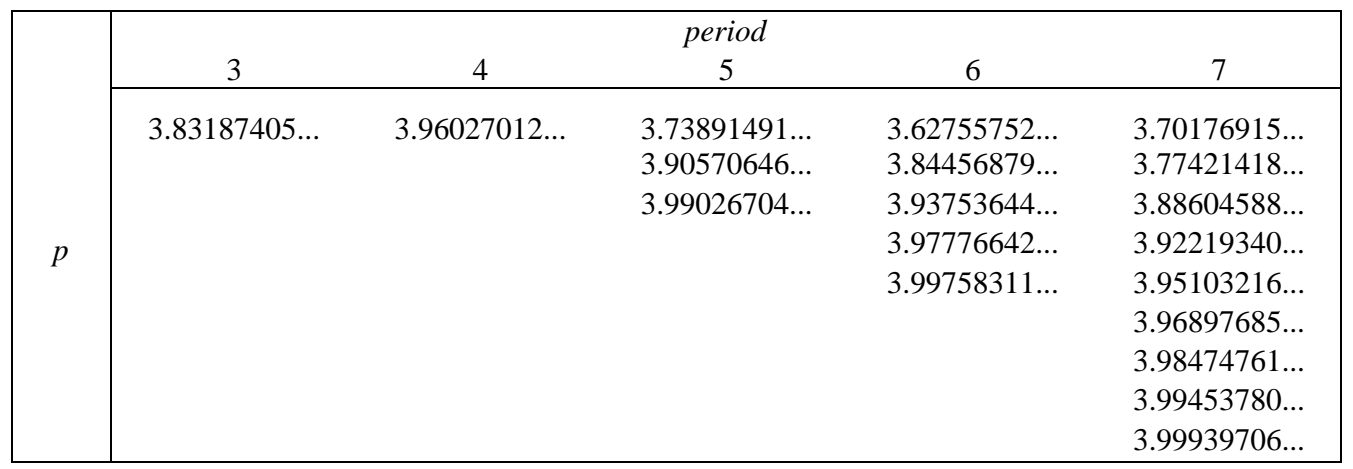

There are 19 parameter values in Table 2 and we could form 171 pairs of parameters $\left(p_{1}, p_{2}\right)$. Except for one single case (which will be discussed later) the system $S^{*}$ obtained with RA algorithm proved to be chaotic and demonstrated sensitive dependence on initial conditions. In all the cases we choose the initial value to be the critical point $z_{0}=0.5$.

For example, let us consider the representative case $\left(p_{1}, p_{2}\right)=(3.83187405,3.96027012)$. The evolution of system $S^{*}$ is drawn in Fig. 1 (the plots represent the time series (a), graphical iteration (b) and histogram (c)). As we can see, the time series and the graphical iteration of system $S^{*}$ seems chaotic and its histogram has an infinity of bars looking chaotic.

Applying the linear surrogate algorithms described by Theiler [11] (Appendix) we provide a trivial confirmation of the fact that the data is not consistent with a linear noise process: we certain do not expect this to be the case. More significantly, we apply the pseudo-periodic surrogate (PPS) algorithm introduced by [12] to eliminate the possibility that the data is either (i) simply periodic, or (ii) consistent with a state dependent noise process. We apply this method to sample trajectories of the chaotification RA algorithm. In order to distinguish between data and surrogates we compute correlation dimension $d_{c}$ (according to the procedure described in [16]) and algorithmic complexity [17]. We use these two statistics because, although correlation dimension is a traditional and popular choice for this purpose, we find algorithmic complexity to be more sensitive. Indeed, computation of algorithmic complexity alone is sufficient to distinguish between the data and standard linear surrogates as well as PPS data. The conclusion is that the observed data is not a monotonic nonlinear transformation of linear noise and is not a noisy periodic orbit. The results of computational complexity calculated for data and surrogates in Fig. 2 confirm the supposition that $S^{*}$ is chaotic.

Figure 2 and the other latter surrogate data figures can be interpreted as follows. The four panels show the results of applying surrogate hypotheses tests for algorithm 0, 1 and 2, and also the PPS algorithm. For these four algorithms the corresponding four hypotheses are (i) independent and identically distributed noise (algorithm 0), (ii) linearly filtered noise (algorithm 1), (iii) a monotonic nonlinear transformation of linearly filtered noise (algorithm 2), and (iv) a noise driven periodic orbit (the PPS algorithm). Each panel depicts a comparison of surrogates and data. For the purposes of this comparison we have generated an ensemble of surrogates consistent with the respective hypothesis (the methods for generating these surrogates are described in the Appendix) and then computed algorithmic complexity for both data and surrogates. The choice of algorithmic complexity (as opposed to any other statistic) is motivated by nothing more than personal preference. Algorithmic complexity (see [17]) measures the complexity (in the sense of how much compression can be achieved with an optimal encoding of the data) of symbolic sequences derived from a time series. The algorithmic complexity therefore depends of the size of the alphabet of that symbolic sequence. This parameter (alphabet size) is illustrated on the abscissa. The exact meaning or method of computation 
of algorithmic complexity is not important for this discussion: it is only a chosen method of comparison of data and surrogates. For each comparison we plot the single algorithmic complexity (as a function of alphabet size) for the data, and the mean and standard deviation of algorithmic complexity of the surrogates. If these two scores differ, then the corresponding hypothesis may be rejected. If the scores do not differ, then no conclusion may be made.

In the case of Fig. 2 the algorithmic complexity is clearly different for data and surrogates for each of algorithm 0, 1 and 2. The conclusion of this is that the data is unlikely to be consistent with a monotonic nonlinear transformation of linearly filtered noise. Although more subtle, the fourth panel also illustrates that the algorithmic complexity for data and surrogates also differs when the surrogates are generated by the PPS algorithm. The conclusion of this calculation is that the data is not consistent with a noisy periodic orbit. Hence, the data contains dynamics that are bounded aperiodic and deterministic. It is consistent with chaos.

We have obtained the Lyapunov exponent of system $S^{*}$ for each one of the 171 pairs of parameter values. In all the cases, with only one exception, which will be treated next, the Lyapunov exponent is positive. Moreover, in each case the power spectrum is typical for chaos: no fundamental harmonics could be found.

According to our measurements, the Lyapunov exponent $\lambda$ of the system $S^{*}$ varies lightly, depending of the array of the $n_{\max }+1$ random values of $p \in \mathcal{P}$. For the considered pair of parameter values $\left(p_{1}, p_{2}\right)=(3.83187405,3.96027012)$ corresponding to the period-3 and period-4 orbits, the Lyapunov exponent of system $S^{*}(0.44,0.53)$ when $n_{\max }=2000$ and it is measured 1000 times. The Lyapunov exponents at $p_{1}$ and $p_{2}$ are obviously $-\infty$, but at these parameter values we can consider two "bridges" with Lyapunov exponents 0.46 and 0.60 respectively (Fig. 3). A graphical estimation of the maximum Lyapunov exponent of the system $S^{*}$ is the mean of the values of the two bridges corresponding to $p_{1}$ and $p_{2}$, that is 0.53 .

The only exception that we have found in the 171 pairs is $\left(p_{1}, p_{2}\right)=(3.83187405,3.84456879)$ that we show in Fig. 4. Note, Fig. 4(b), that these parameter values correspond to period-3 and period- $3 \times 2$ orbits of a same period doubling cascade, and we can consider it as a "consanguineous" case. The Lyapunov exponent of system $S^{*}$ is in the interval $(-0.27,0.0035)$ with a near to zero maximum Lyapunov exponent and a negative minimum Lyapunov exponent. As we can see in Fig. 4(c), the system $S^{*}$ is chaotic but it shows nine very notorious bars. For this cause we can obtain sometimes a positive Lyapunov exponent and sometimes a negative Lyapunov exponent. The power spectrum has a specific form (Fig. 4(d)) and does exhibit a strong peak. But, this does not strictly contradict the hypothesis that the system is chaotic: it only demonstrates that some dominant period is being exhibited. This period may actually be a function of the switching interval between $p_{1}$ and $p_{2}$. Moreover, surrogate data test indicate that the system is not simply periodic: again, the surrogate data test (described above) for data generated from this algorithm also yields the conclusion that the data is not consistent with linear noise or a noisy periodic orbit. We therefore posit chaotic dynamic as the most reasonable alternative. Figure 5 illustrates the result of this calculation. The description and interpretation of the data presented in Fig. 5 is identical to that described above for Fig. 2.

\section{Deterministic anticontrol algorithm}

In the previous section, $p$ was randomly switched between two values of $\mathcal{P}$. In this section we show that if $p_{1}, p_{2} \in \mathcal{P}$ and at each iteration the parameter $p$ alternates in some a deterministically way between $p_{1}$ and $p_{2}$ chaos may rises. The considered deterministic anticontrol algorithm (DA) is 


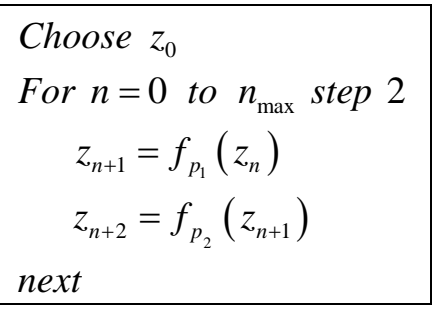

DA algorithm

Let us consider again the logistic map and let $p_{1}$ and $p_{2}$ be two superstable parameter values of Table 2. The DA algorithm generates a system $S^{*}$ that is chaotic the majority of times, but it can be periodic. Using the 171 pairs $\left(p_{1}, p_{2}\right)$ formed with the 19 parameter values of Table 2, we have experimented with DA algorithm using the sequences of parameter values $p_{1_{-} 2}:=p_{1}, p_{2}, p_{1}, p_{2} \ldots$ and $p_{2_{-} 1}:=p_{2}, p_{1}, p_{2}, p_{1} \ldots$. We shall say that the DA algorithm works bi-directionally when it obtains the same results with the two sequences of parameter values $p_{1_{-2}}$ and $p_{2_{-} 1}$. In other words, the algorithm is bi-directional if the system $S^{*}$ is chaotic with both sequences or it is periodic with both sequences. Some few times the system $S^{*}$ is chaotic using one of the sequences and it is periodic using the other. We resume the obtained results with the 171 pairs in Table 3.

\begin{tabular}{|c|c|}
\hline Behavior & Cases number \\
\hline Bi-directionally chaotic & 161 \\
\hline Bi-directionally periodic & 7 \\
\hline Chaotic $\left(p_{1 \_2}\right)$ and periodic $\left(p_{2 \_1}\right)$ & 3 \\
\hline
\end{tabular}

Note that the system $S^{*}$ clearly is more bi-directionally chaotic (94\%) than bi-directionally periodic (4\%). Rarely (2\%) the system $S^{*}$ is chaotic (with one of the sequences of parameter values) and periodic (with the other sequence). Actually, these rare chaotic and periodic cases can be considered as bi-directionally chaotic ones because the periodicity only occurs for a very narrow interval of initial values centered at $z_{0}=0.5$. Table 4 shows the 7 pairs of parameter values that cause a bi-directionally periodic behavior and the 3 pairs of parameter values that cause a chaotic $\left(p_{1_{-} 2}\right)$ and periodic $\left(p_{2_{1} 1}\right)$ behavior. By $\lambda_{1 \_2}$ and $\lambda_{2_{-} 1}$ we denote the Lyapunov exponents corresponding to sequences $p_{1_{-2}}$ and $p_{2 \_1}$ respectively. 
Table 4

Pairs of parameter values of the $10 S^{*}$ systems of Table 2 with non-chaotic behavior.

\begin{tabular}{|lccc|}
\hline \multicolumn{1}{|c}{ Behavior } & $\left(p_{1}, p_{2}\right)$ & $\lambda_{1 \_2}$ & $\lambda_{2 \_}$ \\
\hline Bi-directionally periodic & $(3.62755752,3.70176915)$ & -0.27 & -0.27 \\
Bi-directionally periodic & $(3.62755752,3.73891491)$ & -0.11 & -0.11 \\
Bi-directionally periodic & $(3.62755752,3.99939706)$ & -0.021 & -0.018 \\
Bi-directionally periodic & $(3.73891491,3.83187405)$ & -0.97 & -0.96 \\
Bi-directionally periodic & $(3.92219340,3.96027012)$ & -0.17 & -0.17 \\
Bi-directionally periodic & $(3.93753644,3.96027012)$ & -0.020 & -0.024 \\
Bi-directionally periodic & $(3.95103216,3.96027012)$ & -0.16 & -0.16 \\
Chaotic $\left(p_{1_{2}}\right)$ and periodic $\left(p_{2 \_1}\right)$ & $(3.62755752,3.90570646)$ & +0.220 & -0.063 \\
Chaotic $\left(p_{1_{2}}\right)$ and periodic $\left(p_{2_{1} 1}\right)$ & $(3.62755752,3.95103216)$ & +0.19 & -0.18 \\
Chaotic $\left(p_{1_{-2}}\right)$ and periodic $\left(p_{2_{1} 1}\right)$ & $(3.62755752,3.97776642)$ & +0.21 & -0.04 \\
\hline
\end{tabular}

Let us consider one of the 161 pairs with a bi-directionally chaotic behavior, for example $\left(p_{1}, p_{2}\right)=(3.70176915,3.93753644)$ (Fig. 6). Note that the time series, graphical iteration and histogram of the system $S^{*}$ are chaotic with the sequence $p_{1_{-} 2}$. The same occurs with the sequence $p_{2_{-} 1}$. The Lyapunov exponents are $\lambda_{1_{-} 2}=\lambda_{2_{-1}}=0.44$ indicating that $S^{*}$ is chaotic. We have repeated the surrogate tests describe above. The results (precisely analogous to those presented in Figs. 2 and 5) against each of the four hypotheses are presented in Fig. 7. For each case, the result is the same: the surrogate tests indicate that this data is inconsistent with either general linear noise (a static monotonic nonlinear transformation of linearly filtered noise) or a noisy periodic orbit. The most likely alternative hypothesis is deterministic chaos.

Finally, let us consider one of the three pairs with chaotic and periodic behavior, for example $\left(p_{1}, p_{2}\right)=(3.62755752,3.97776642)$ (Figs. 8-11). Let $z_{0}=0.5$ be the initial point of system $S^{*}$. Firstly, as we can see in Fig. 8, the system $S^{*}$ is chaotic with the sequence of parameter values $p_{1_{-2}}$. The surrogate tests (Fig. 9) indicate that this data is inconsistent with either general linear noise (a static monotonic nonlinear transformation of linearly filtered noise) or a noisy periodic orbit. The most likely alternative hypothesis is deterministic chaos. Secondly, as we can see in Fig. 10, the system is periodic with the sequence of parameter values $p_{2_{-} 1}$. The surrogate tests are shown in Fig. 11. Actually, the periodicity in this case is only approximate. Although both frequency and surrogate analysis support the conclusion of periodicity (the surrogate conclusion is marginal and depends on the choice of encoding scheme utilized by algorithmic complexity), a much closer examination contradicts this finding. Figure 12 illustrates the delay embedding achieved when embedding the data in two dimensions with a time delay of 10 (corresponding exactly to the supposed period). As one would expect for a periodic time series (with period 10), the result is 10 accumulation points on the identity line. However, closer examination of any of these points reveals a more subtle phenomenon. In Fig. 12 we zoom into two of these accumulation points and observe bounded aperiodic deterministic dynamics: chaos. The same result (not shown here) is observed for each of the other points. Of course, the scale of this feature (as is attested by the extent of the respective axes) is very fine. Any numerical error or experimental noise of a larger scale will obliterate this feature. This chaotic and periodic behavior only occurs when the initial value is in the narrow interval $[0.4998,0.5002]$ centered at the value $z_{0}=0.5$. When the initial value is out of this interval the system $S^{*}$ has a bi-directionally chaotic behavior. 


\section{Concluding remarks}

We present here two anticontrol algorithms belonging to the class of anticontrol which act on the control parameters. We have shown that both the random combination and the deterministic (periodic) combination of two periodic dynamics of the form $p x(1-x)$ results in a chaotic dynamics. This "order + order $=$ chaos" phenomenon is analogous to the original Parrondo's paradox and is obtained by exploiting Parrondo's philosophy of alternation.

Proving analytically that the chaos so generated is indeed a "true" chaos in a rigorous mathematical sense remains a task for a future work. It goes without saying that the two periodic dynamics considered in Section 3 can be combined in other deterministic manners (for example $\left.p_{1}, p_{1}, p_{2}, p_{1}, p_{1}, p_{2} \ldots\right)$. Whether paradox occurs or not in all these cases remains an open problem.

The use of other random distributions, different to that utilized in this paper (uniform distribution), represents a possible interesting approach. In this manner the random influence of the parameters $p_{1}$ and $p_{2}$ could be realized with different weights.

The generalization for more than two values for the control parameter $p$ can be easily made.

\section{Appendix: Surrogate tests applied in Section 2}

The basic principal of surrogate data hypothesis testing is to determine whether a given observed time series is consistent with a specific hypothesis by generating an ensemble of data and making a qualitative comparison between them. For example, to determine whether an observed time series is consistent with (say) the hypothesis of random i.i.d (independent and identically distributed) noise, one generates an ensemble of surrogate data sets that are i.i.d. noise. The trick is to generate this ensemble in such a way that they are also typical of the original data. For the case of the null hypothesis of i.i.d. noise, this can be achieved by shuffling the original data: each shuffle of the original data preserves the rank distribution (here, "the histogram") of the data, but destroys temporal ordering: precisely the property one expects to be absent for i.i.d. noise. Having generated the ensemble, one can then compute some test statistic (in the current paper we have used algorithmic complexity, and this is discussed more fully elsewhere) for both the data and the ensemble of surrogates. If the value of this statistic for the data is atypical of the distribution obtained from the surrogates, then the associated null hypothesis may be rejected. Otherwise, it may not.

The three algorithms to test the three hypotheses of i.i.d. noise, linearly filtered noise and a monotonic nonlinear transformation of linearly filtered noise may be described (briefly) as follows. To test for i.i.d. noise (algorithm 0 ) the surrogate is generated by simply randomly re-ordering the data. To test for linear noise (algorithm 1) the phases of the Fourier transform of the data are randomised (or even just re-ordered) and the inverse Fourier transform is the corresponding surrogate.

The procedure to test for a monotonic nonlinear transformation of linearly filtered noise is a little more complex. First, the data is scaled so that it is Gaussian rank-distributed (or, equivalently, Gaussian noise is re-ordered to have the same rank distribution as the data). The Gaussian rankdistributed (but not i.i.d.) signal is then Fourier transformed and its phases randomised (that is, we take an algorithm 1 surrogate of the Gaussian rank-distributed signal). Finally, the data is re-ordered to have the same rank distribution as the algorithm 1 surrogate of the Gaussian rank-distributed signal.

As it is well known, surrogate data methods are designed to provide a Monte-Carlo test of a specific null hypothesis from a single observed time series [13]. Comparison of the data and an ensemble of surrogates will either indicate that the data is atypical of the surrogates (and therefore the underlying null hypothesis), or that it is not. Whereas the standard linear surrogate tests test the hypotheses that the data is independent noise, linear noise, or nonlinearly filtered linear noise, the PPS algorithm introduced by [12] tests a more sophisticated hypothesis.

The procedure, described in detail elsewhere, can be briefly described as follows. One first embeds the original time series, and then generates surrogates by choosing an initial point on the embedded attractor and iterating it. At each iteration one randomly chooses a neighbour of the current state 
(possibly the current state itself) and then the next point is the temporal successor of that chosen state. The effect of this procedure is that one generates a random trajectory (at each step there is a random dynamic noise perturbation to the trajectory) following the same general nonlinear structure as the original system. The surrogate is a noisy periodic orbit. Therefore, if the original system is a noisy periodic orbit the data and surrogates are indistinguishable. If the original system is a deterministic bounded and aperiodic system, then the data and surrogates will be distinct. This was demonstrated in [12].

\section{Acknowledgements}

Miguel Romera is supported by Ministerio de Educación y Ciencia (MEC), Spain, Research Grant SEG 2004-02418. Michael Small is supported by Hong Kong University Grants Council Competitive Earmarked Research Grant (CERG) No. PolyU 5235/03E. Marius-F. Danca acknowledges the Centre for Chaos Control and Complex Networks at the City University of Hong Kong for providing him a visiting research fellowship in 2007.

\section{References}

[1] G. Chen and Y. Shi, Introduction to anticontrol of discrete chaos: theory and applications, Philos. T. Roy. Soc. A 364:2433-2447 (2006)

[2] G. Chen and D. Lai, Feedback anticontrol of discrete chaos, Int. J. Bifurcat. Chaos 8: 1585-1590 (1998).

[3] A. Vanecek and S. Celikovsky, Chaos synthesis via root locus, IEEE T. Circuits-I 41:59-60 (1994).

[4] X. F. Wang and G. Chen, Chaotification via arbitrarily small feedback controls: Theory, method, and applications, Int. J. Bifurcat. Chaos 10:549-570 (2000).

[5] M-F. Danca, Chaotifying discontinuous dynamical systems via time-delay feedback algorithm, Int. J. Bifurcat. Chaos 14:2321-2339 (2004).

[6] G. Chen, Chaos: control and anticontrol, IEEE Circuits Syst. Soc. Newslett. 9:1-5 (1998).

[7] F.X. Witkowski, L.J. Leon, P.A. Penkoske, W.R. Giles, M.L. Spano, W.L. Ditto and A.T. Winfree, Spatiotemporal evolution of ventricular fibrillation, Nature 392:78-82 (1998).

[8] J.M.R. Parrondo, G.P. Harmer and D. Abbott, New paradoxical games based on Brownian ratchets, Phys. Rev. Lett. 85:5226-5229 (2000).

[9] G.P. Harmer and D. Abbot, Parrondo’s Paradox, Stat. Sci. 14:206-213 (1999).

[10] J. Almeida, D. Peralta-Salas and M. Romera, Can two chaotic systems rise to order? Physica D 200:124-132 (2005).

[11] J. Theiler, S. Eubank, A. Longtin, B. Galdrikian and J. Doyne, Testing for nonlinearity in time series: The method of surrogate data, Physica D 58:77-94 (1992).

[12] M. Small , D. Yu and R.G. Harrison, Surrogate test for pseudoperiodic time series data, Phys. Rev. Lett. 87:188101 (2001).

[13] M. Small, T. Nakamura and X. Luo, Surrogate data methods for data that isn't linear noise. In New Research on Nonlinear Phenomena, Nova Science Publishers, in press.

[14] F. Morrison, Predictability and forecasting, Proceedings of the 1985 International Conference of the System Dynamics Society, The System Dynamics Society, Keystone, CO, 565-574, 1985.

[15] J. Honerkamp, Stochastic dynamical systems, Concepts, Numerical Methods, Data Analysis, VCH, New York, 1994.

[16] D.J. Yu, M. Small, R.G. Harrison and C. Diks, Efficient implementation of the Gaussian kernel algorithm in estimating invariants and noise level from noisy time series data, Phys. Rev. E 61:3750-3756 (2000).

[17] M. Small, Applied Nonlinear Time Series Analysis: Applications in Physics, Physiology and Finance, Nonlinear Science Series A 52, World Scientific, 2005. 


\section{Figure captions}

Fig. 1. The system $S^{*}$ obtained with the RA algorithm and the pair of parameter values $\left(p_{1}, p_{2}\right)=(3.83187405$, 3.96027012) . (a) Time series; (b) Graphical iteration; (c) Histogram.

Fig. 2. The figure depicts the results of the surrogate data testing for the data generated from $\left(p_{1}, p_{2}\right)=(3.83187405$, 3.96027012). The four panels, from top-left to bottom right test the four hypotheses of: (i) independent and identically distributed noise; (ii) linearly filtered noise; (iii) a monotonic nonlinear transformation of linearly filtered noise; and, (iv) a noisy periodic orbit (the PPS algorithm). For each panel the mean and standard deviation of 50 surrogates (solid line and error bars) together with the corresponding values for the data (solid line). This computation is repeated for a range of embedding dimensions (from 2 to 10, presented on the horizontal axis), and, the statistic shown on the vertical axes is normalised complexity, as described in the main text. In each case, for each of the four tests, the null hypothesis may be rejected: the data is not consistent with either linear noise (including independent and identically distributed noise or a monotonic nonlinear transformation of linear noise) or a noisy periodic orbit.

Fig. 3. Lyapunov exponent of the logistic map as a function of the parameter value. Note the two "bridges" in $p_{1}$ and $p_{2}$.

Fig. 4. The "consanguineous" case obtained with the RA algorithm and the pair of parameter values $\left(p_{1}, p_{2}\right)=(3.83187405$, 3.84456879) . (a) Time series; (b) Graphical iteration; (c) Histogram; (d) Power spectrum.

Fig. 5. Results of the surrogate test for $\left(p_{1}, p_{2}\right)=(3.83187405,3.84456879)$. The interpretation of these results is the same as Fig. 2.

Fig. 6. The system $S^{*}$ obtained with the DA algorithm, the pair of parameter values $\left(p_{1}, p_{2}\right)=(3.70176915,3.93753644)$ and the sequence $p_{1 \_2}$. (a) Time series; (b) Graphical iteration; (c) Histogram.

Fig. 7. Results of the surrogate test for $\left(p_{1}, p_{2}\right)=(3.70176915,3.93753644)$ and the sequence $p_{2_{-} 1}$. The interpretation of these results is the same as Fig. 2.

Fig. 8. The system $S^{*}$ obtained with the DA algorithm, the pair of parameter values $\left(p_{1}, p_{2}\right)=(3.62755752,3.97776642)$ and the sequence $p_{1_{-} 2}$. (a) Time series; (b) Graphical iteration; (c) Histogram.

Fig. 9. Results of the surrogate test for $\left(p_{1}, p_{2}\right)=(3.62755752,3.97776642)$ and the sequence $p_{1 \_2}$. The interpretation of these results is the same as in Fig. 2.

Fig. 10. The system $S^{*}$ obtained with the DA algorithm, the pair of parameter values $\left(p_{1}, p_{2}\right)=(3.62755752,3.97776642)$ and the sequence $p_{2_{-} 1}$. (a) Time series; (b) Graphical iteration; (c) Histogram.

Fig. 11. Results of the surrogate test for $\left(p_{1}, p_{2}\right)=(3.62755752,3.97776642)$ and the sequence $p_{2_{-} 1}$. The interpretation of these results is the same as Fig. 2.

Fig. 12. Delay embedding for $\left(p_{1}, p_{2}\right)=(3.62755752,3.97776642)$ and the sequence $p_{2_{1}}$. A delay embedding (with delay 10 , corresponding to the supposed period) is illustrated. A zoom into each of the accumulation points illustrates that some fine underlying chaotic modulation of the periodic signal (only two such zooms are illustrated here). The bounding boxes (maximum to minimum value) on the accumulation points at 0.2997 and 0.6464 are $1 \times 10^{-4}$ and $3 \times 10^{-5}$ respectively. 


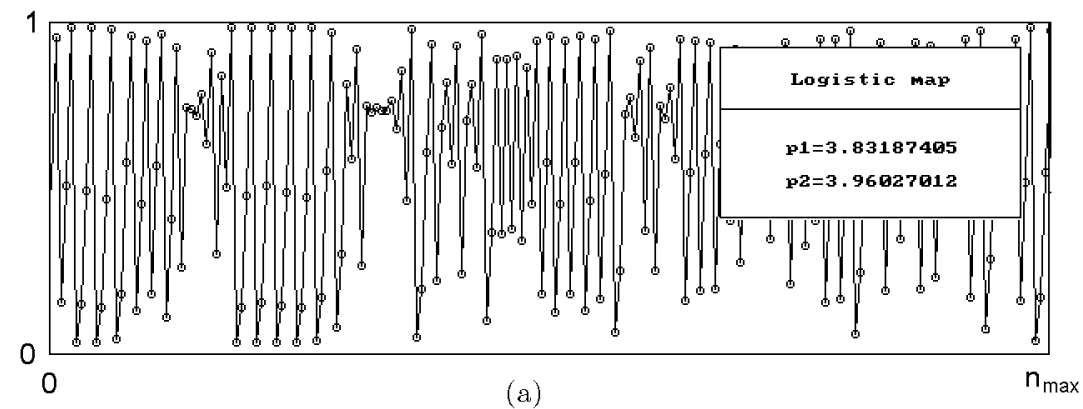

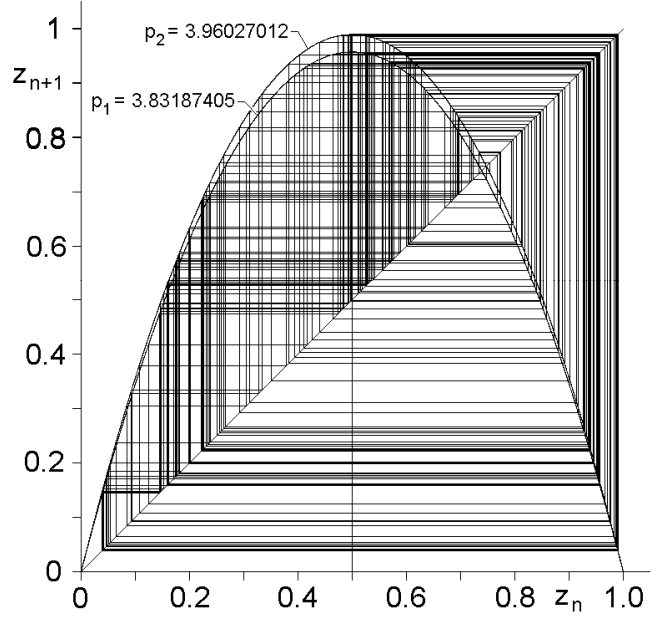

(b)

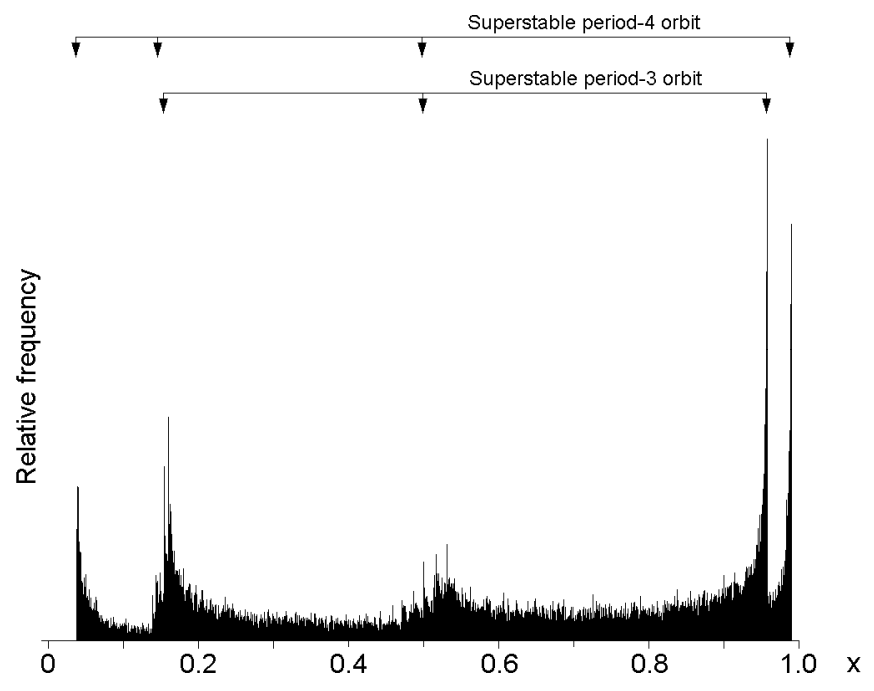

(c)

Fig. 1 
Alg. 0: data1

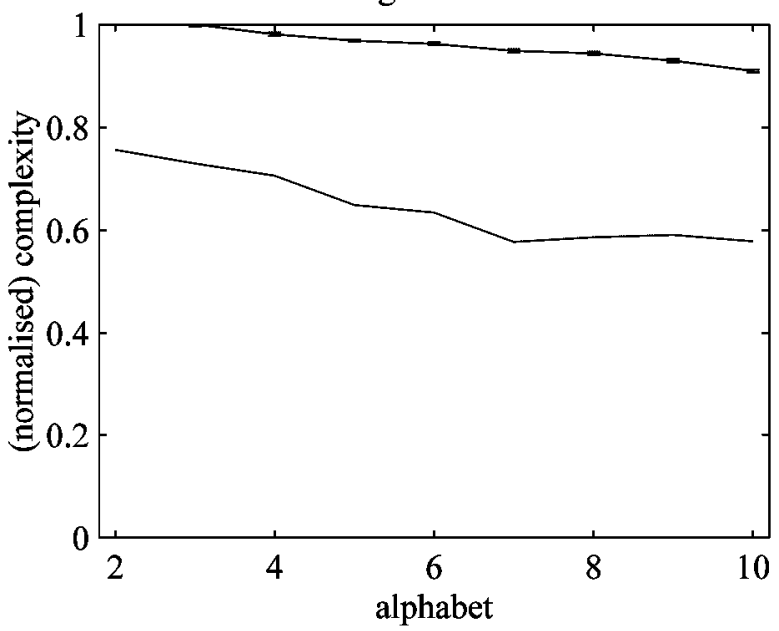

Alg. 2: data1

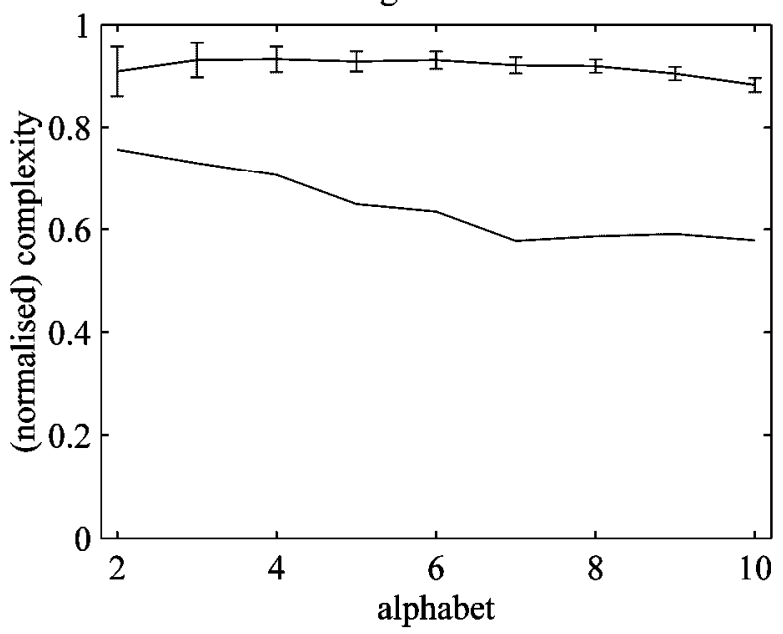

Alg. 1: data1

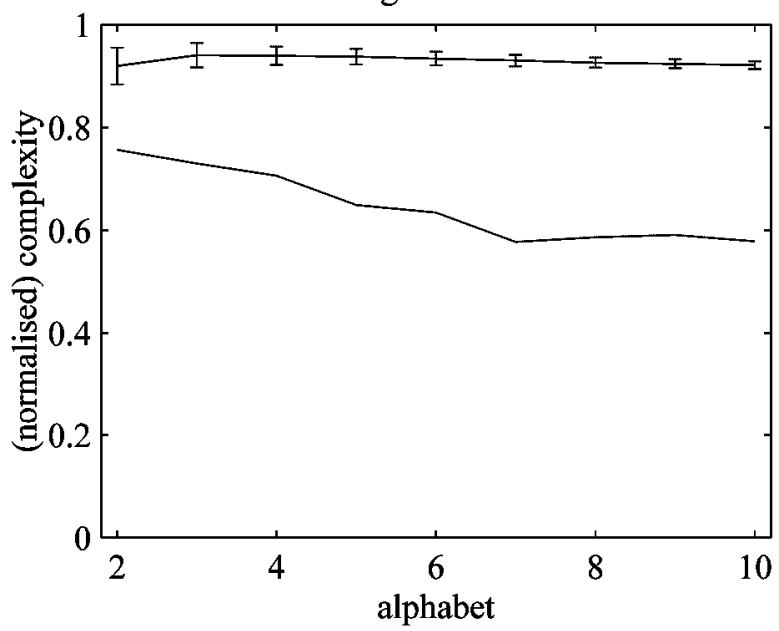

PPS: data1

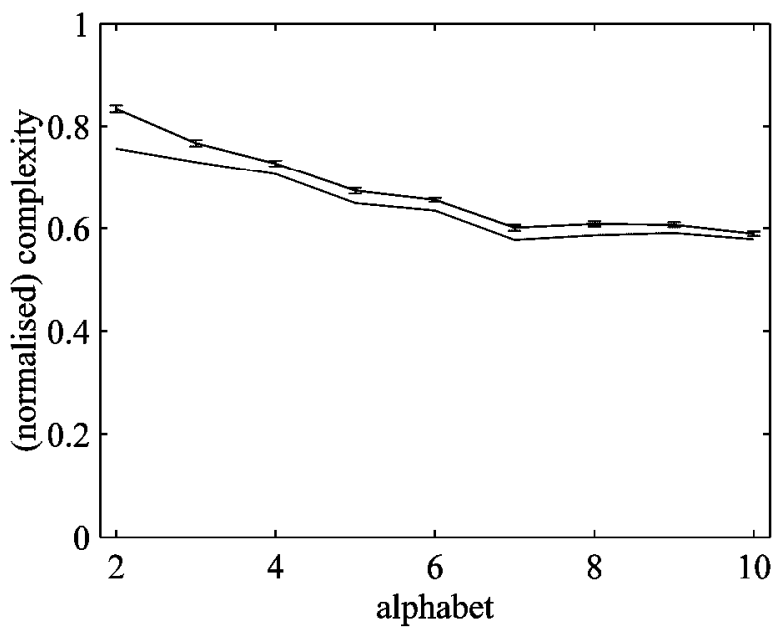

Fig. 2 


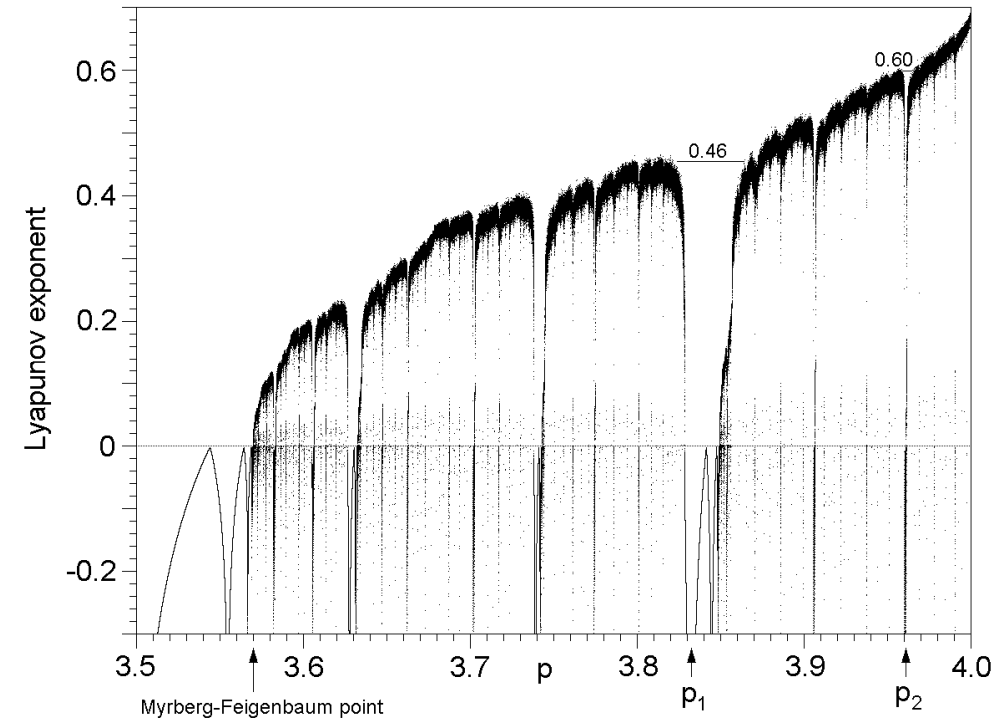

Fig. 3 


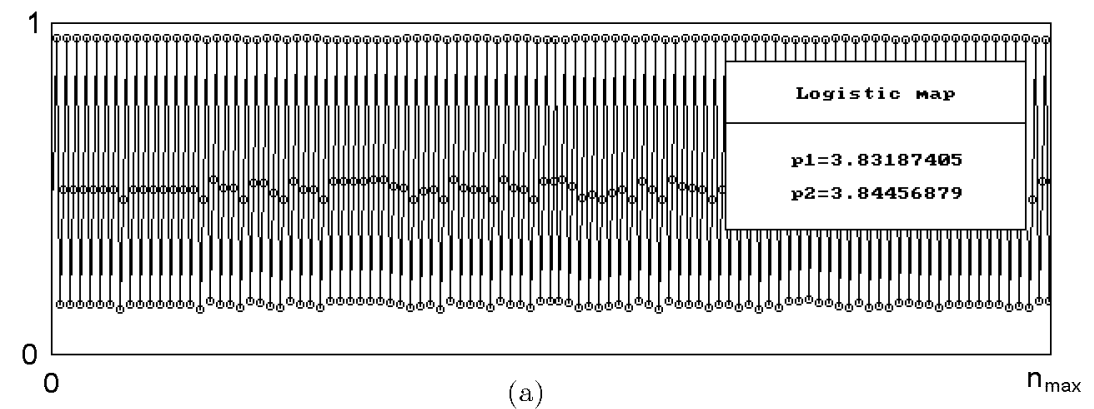

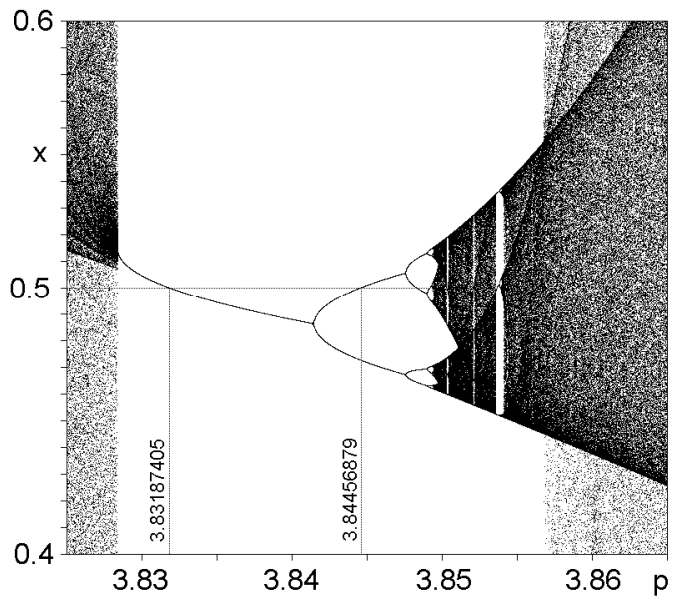

(b)

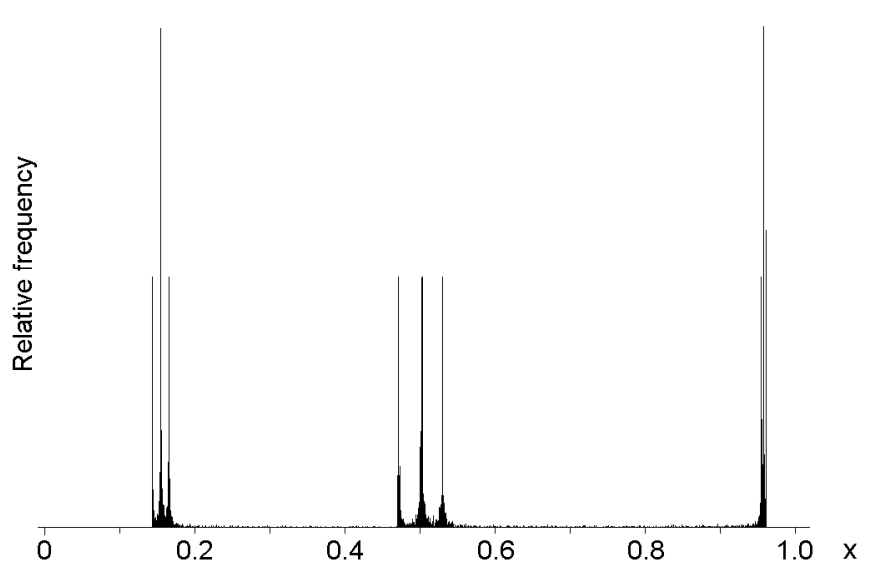

(c)

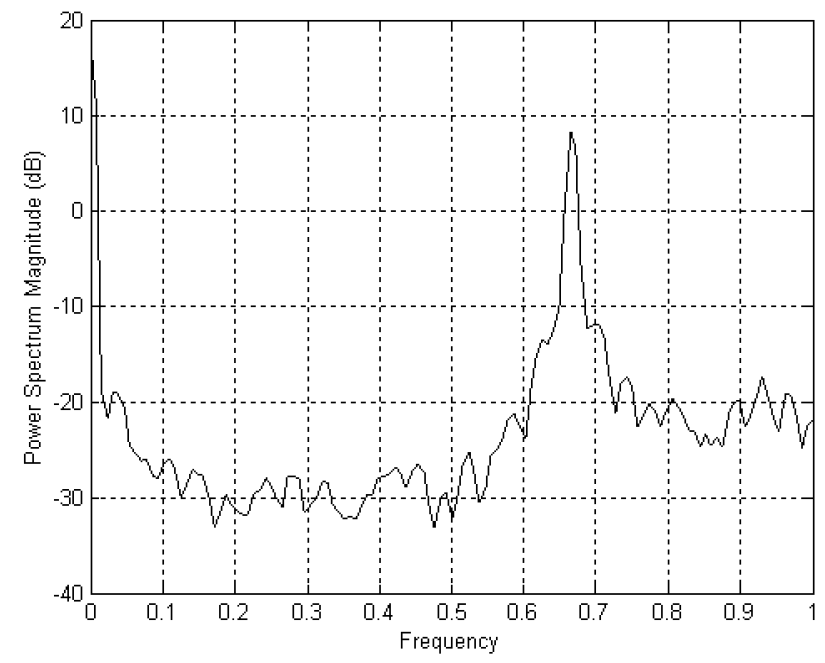

(d)

Fig. 4 
Alg. 0: data2

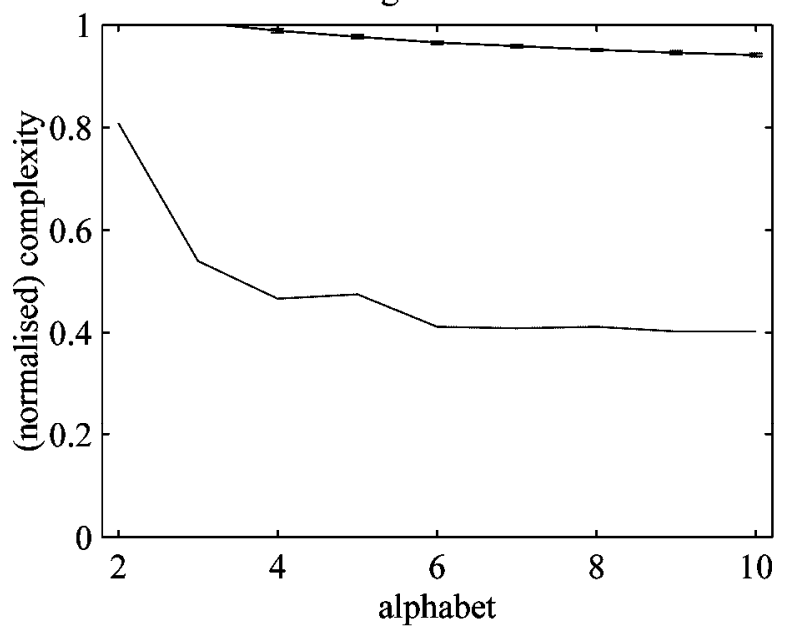

Alg. 2: data2

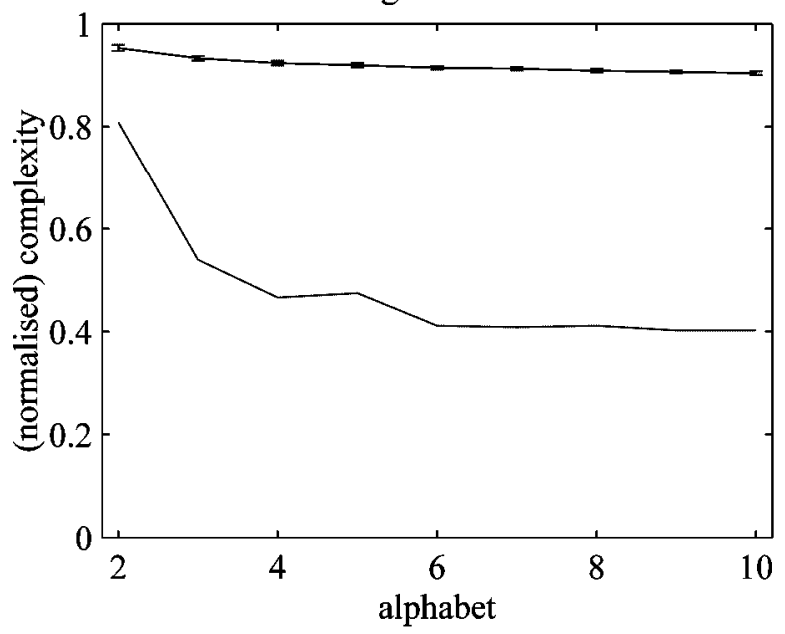

Alg. 1: data2

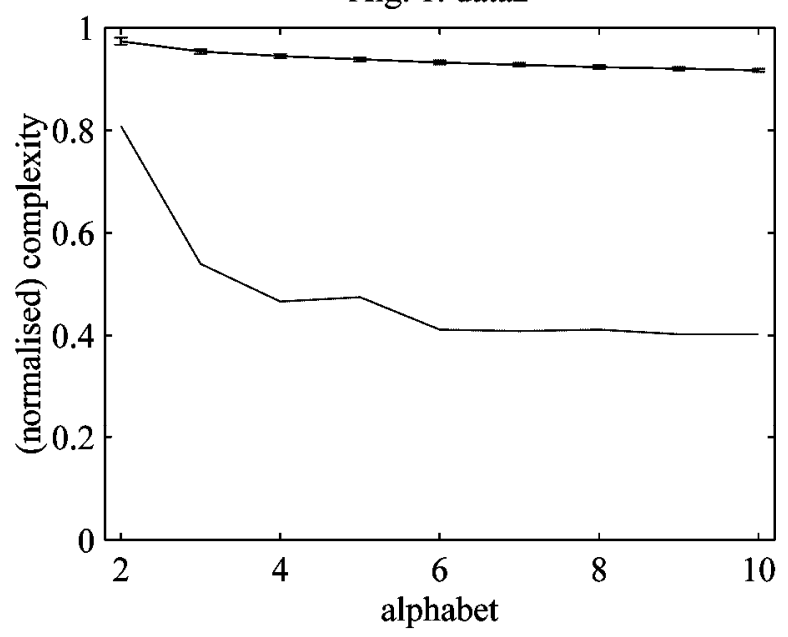

PPS: data2

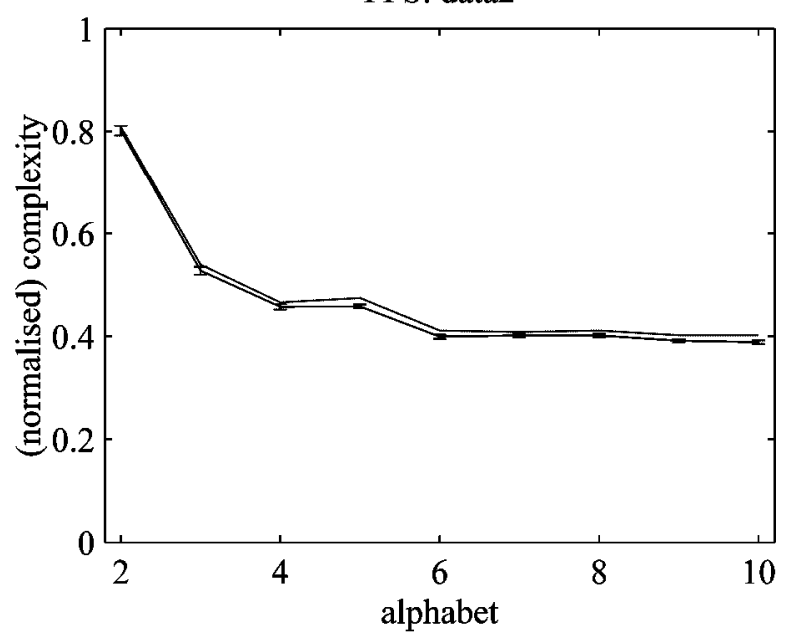

Fig. 5 


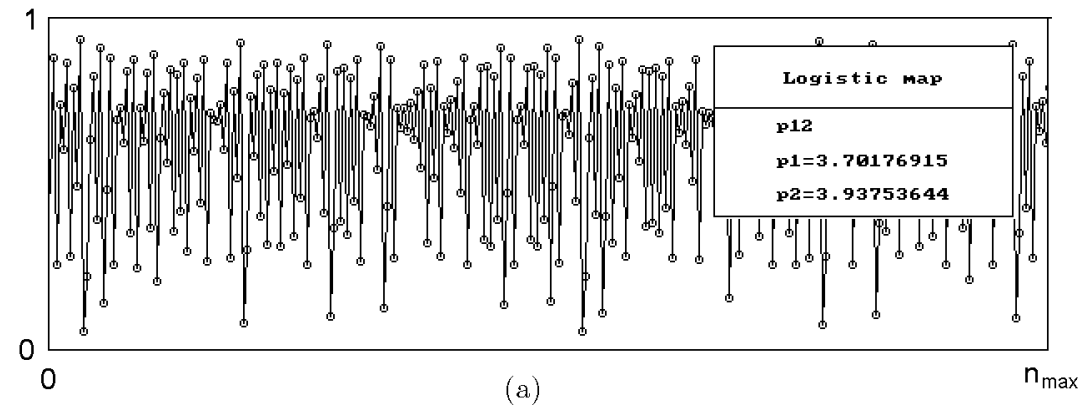

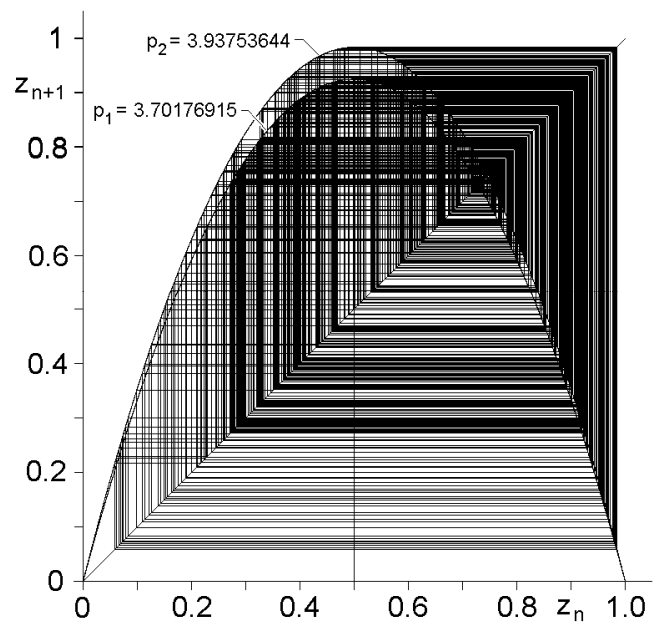

(b)

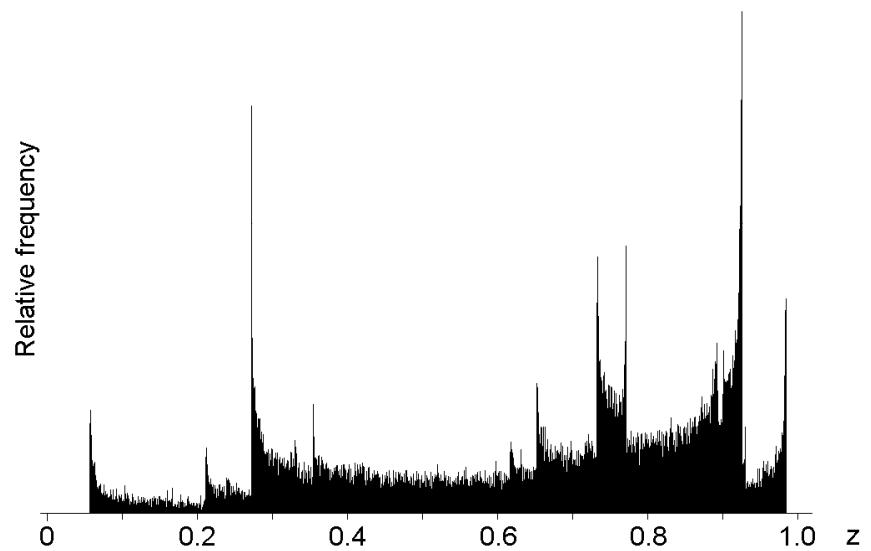

(c)

Fig. 6 
Alg. 0: data9

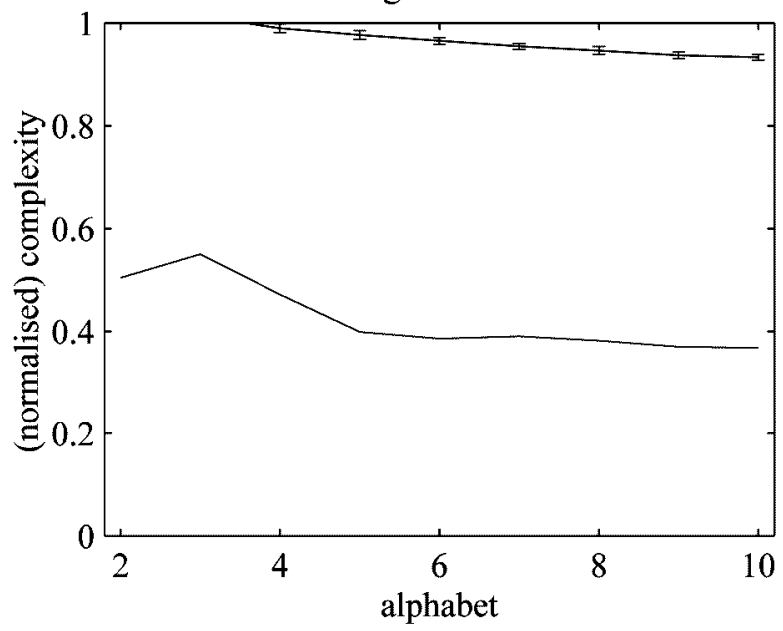

Alg. 2: data9

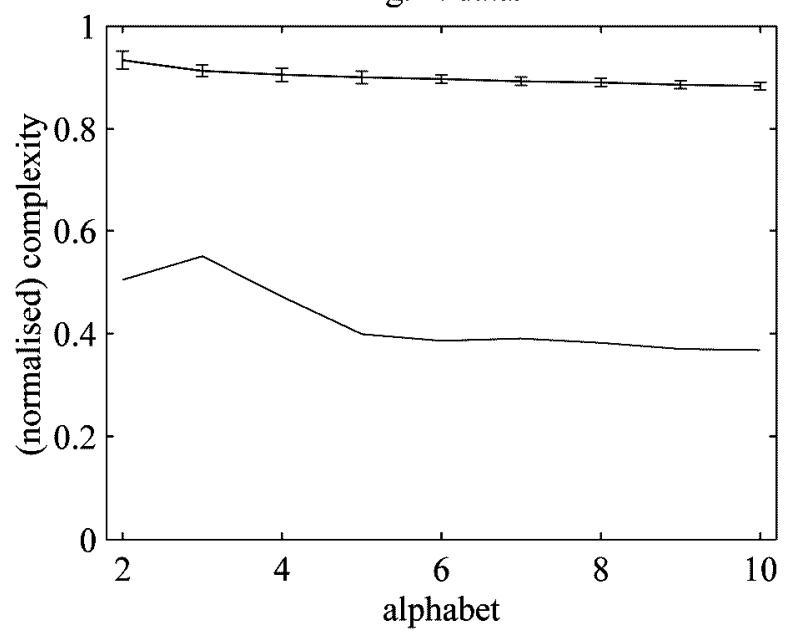

Alg. 1: data9

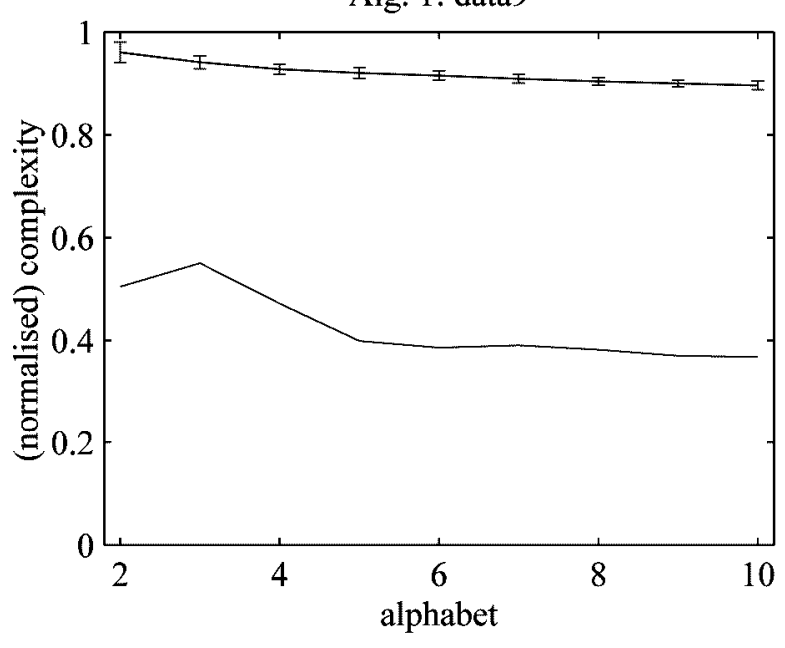

PPS: data9

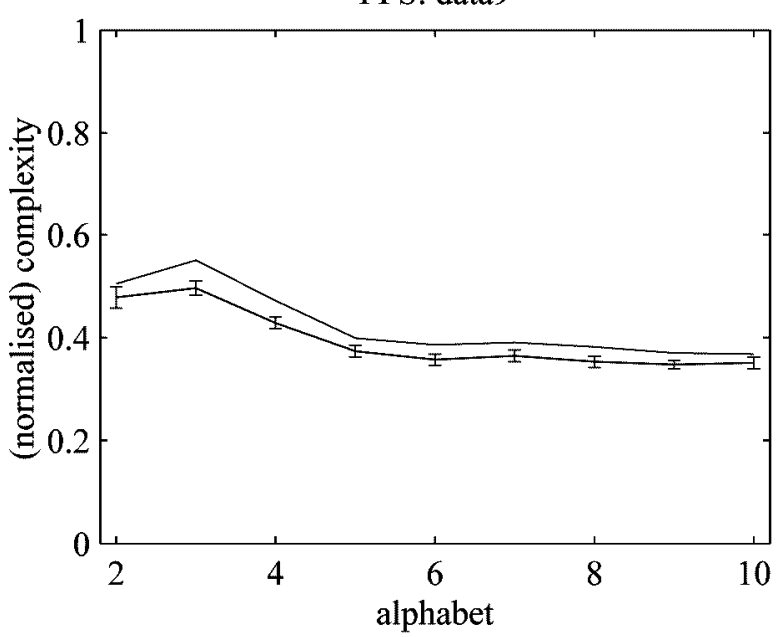

Fig. 7 


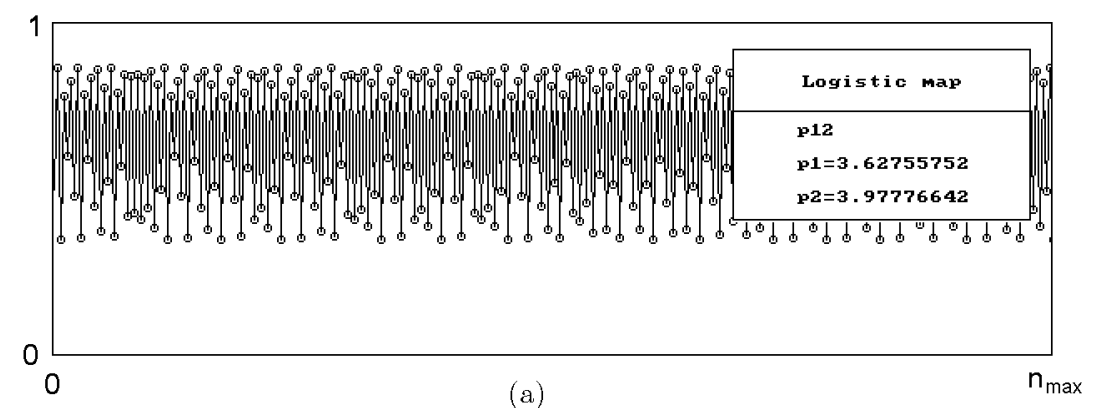

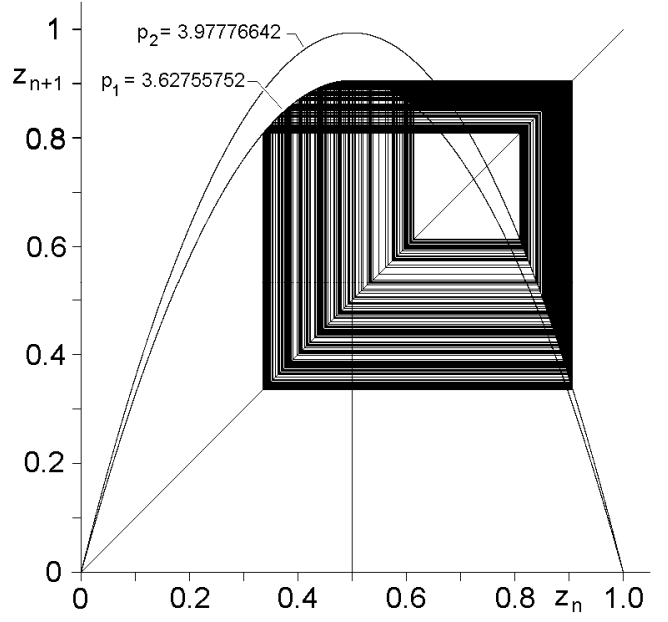

(b)

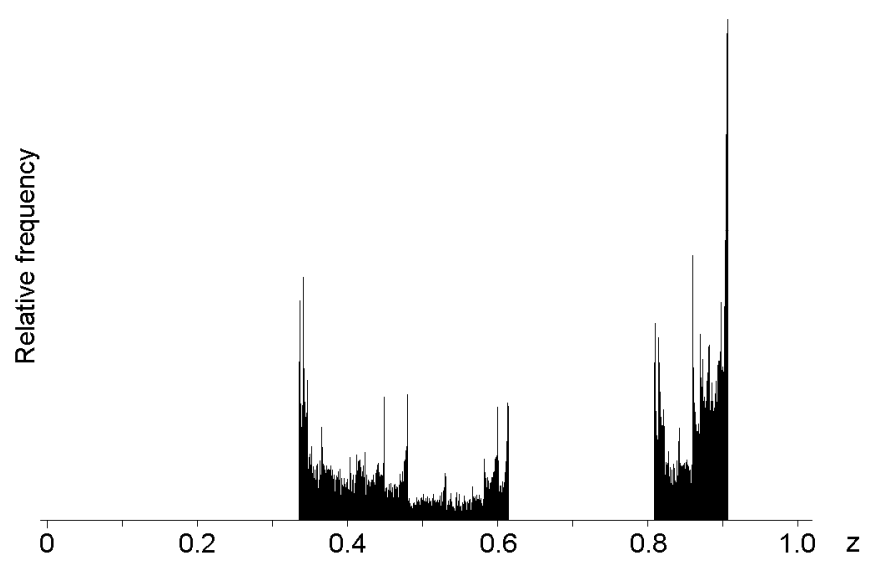

(c)

Fig.8 
Alg. 0: data10

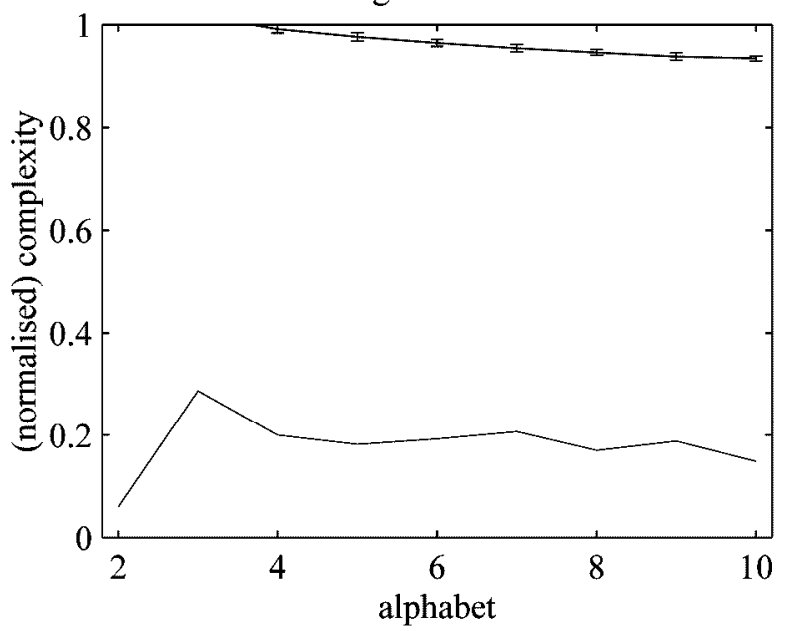

Alg. 2: data10

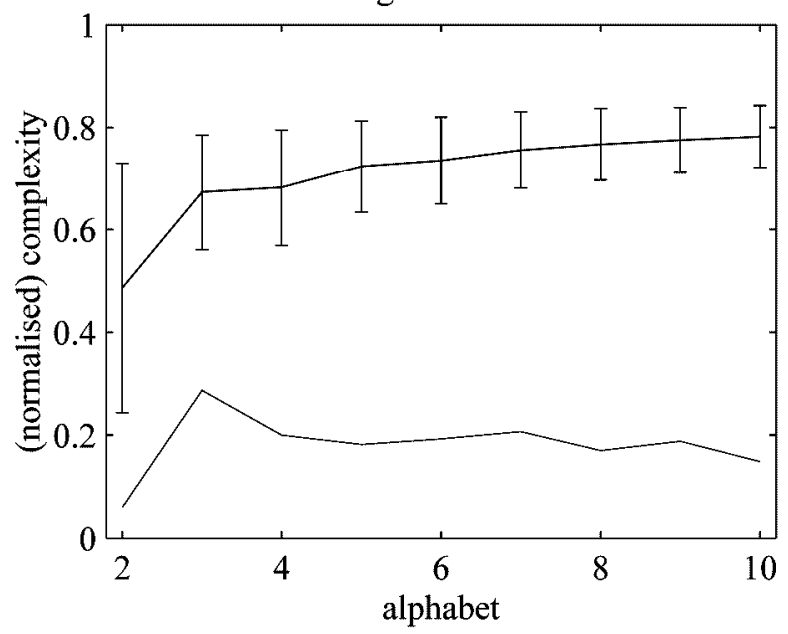

Alg. 1: data10

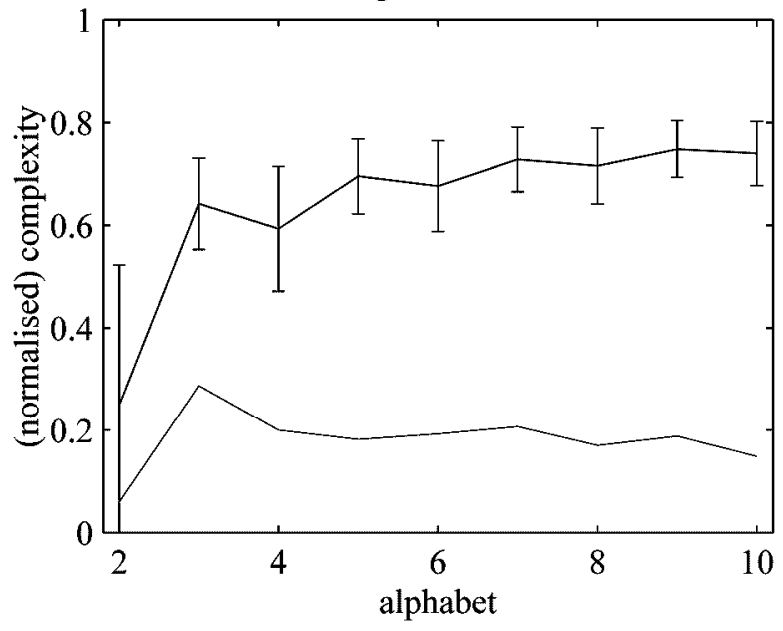

PPS: data10

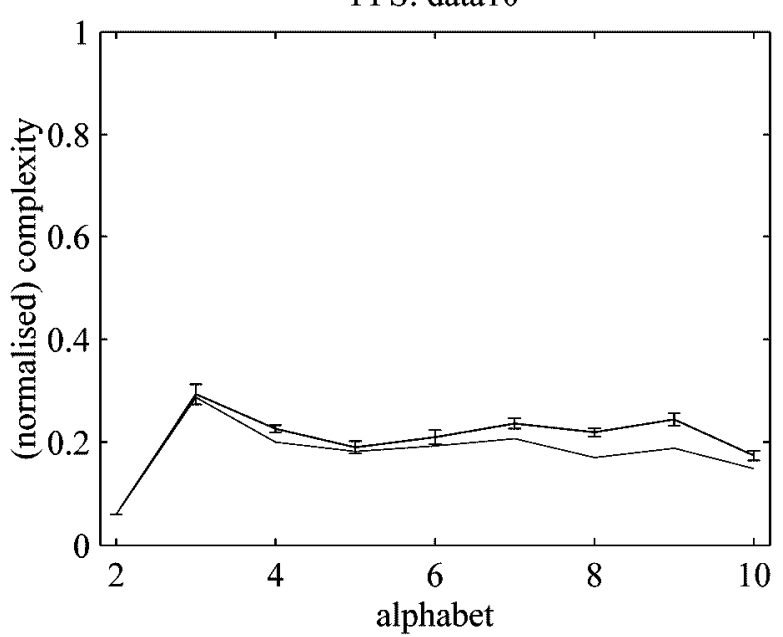

Fig. 9 


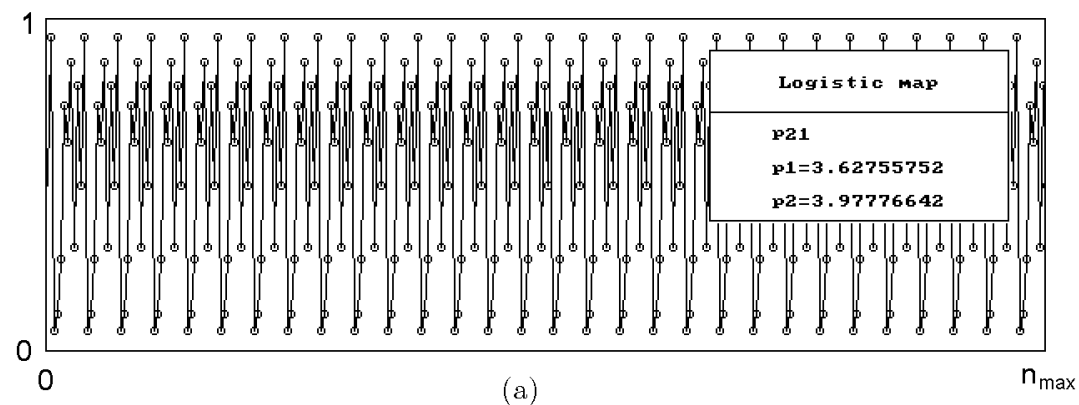

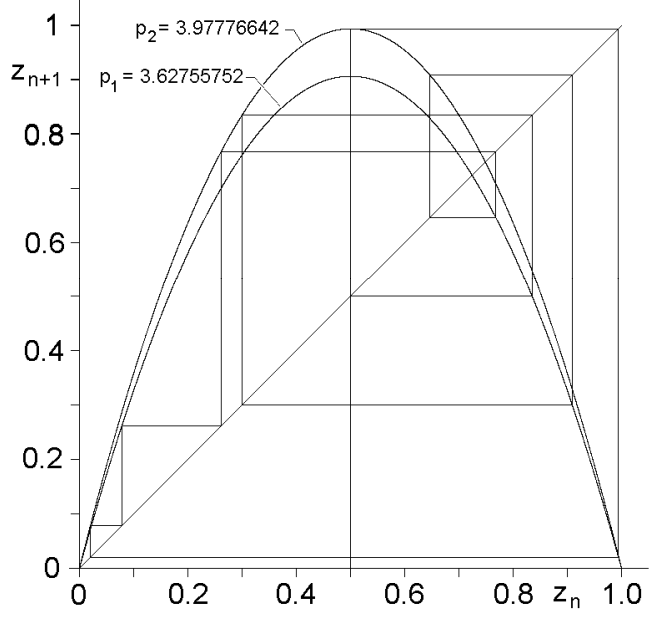

(b)

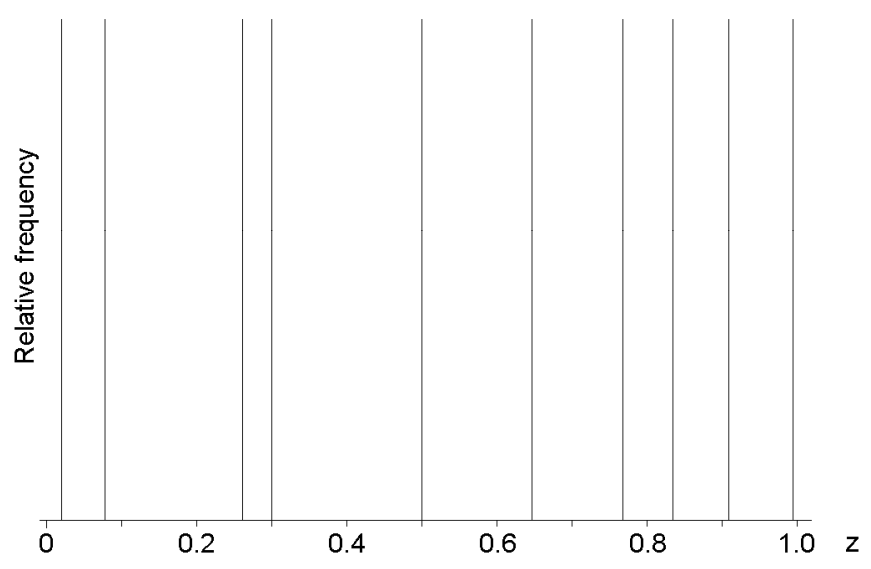

(c)

Fig. 10 
Alg. 0: data11

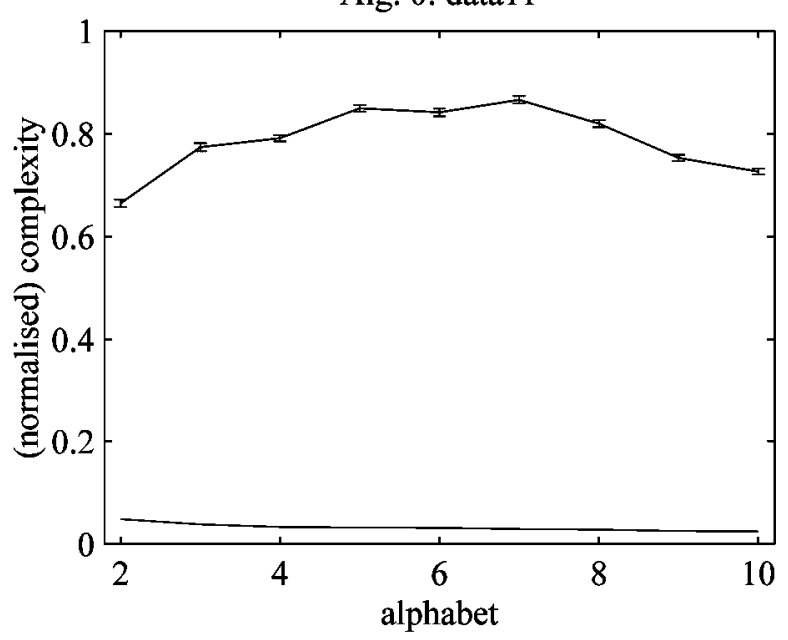

Alg. 2: data11

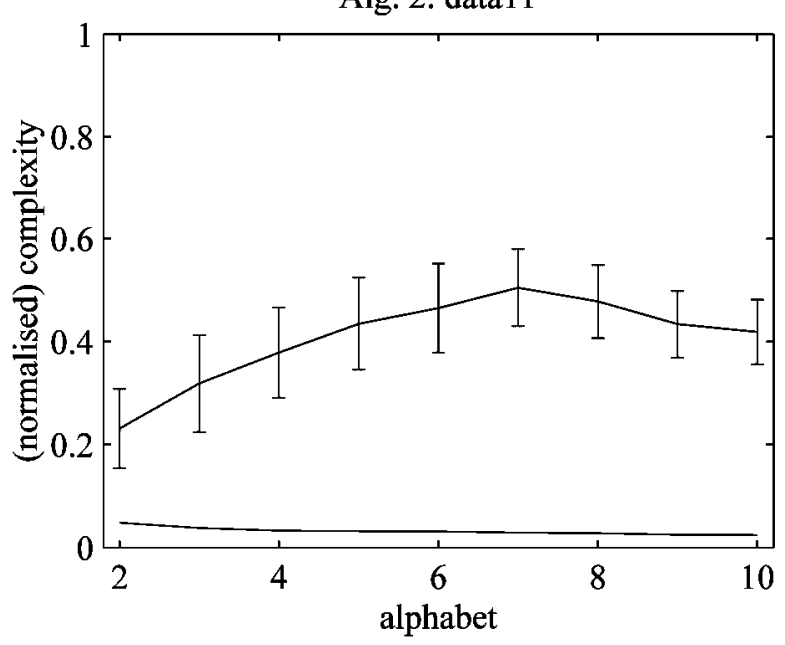

Alg. 1: data11

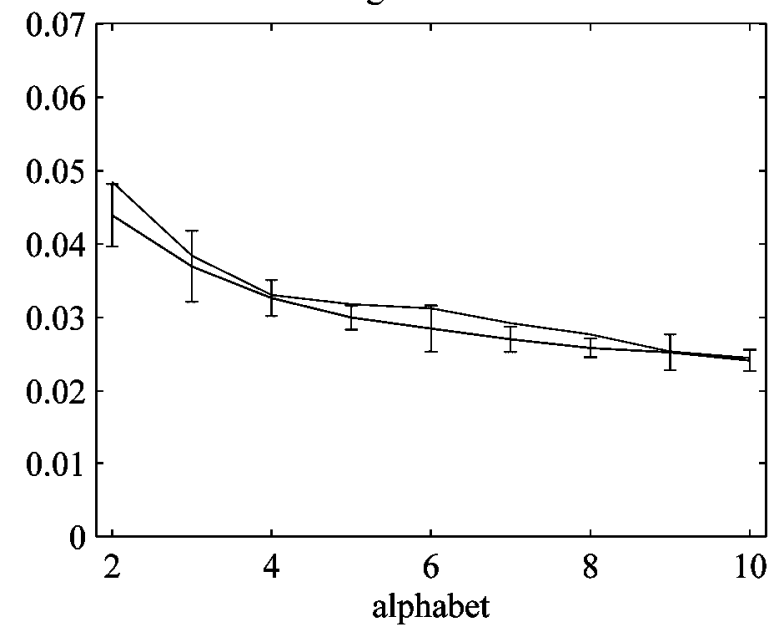

PPS: data1 1

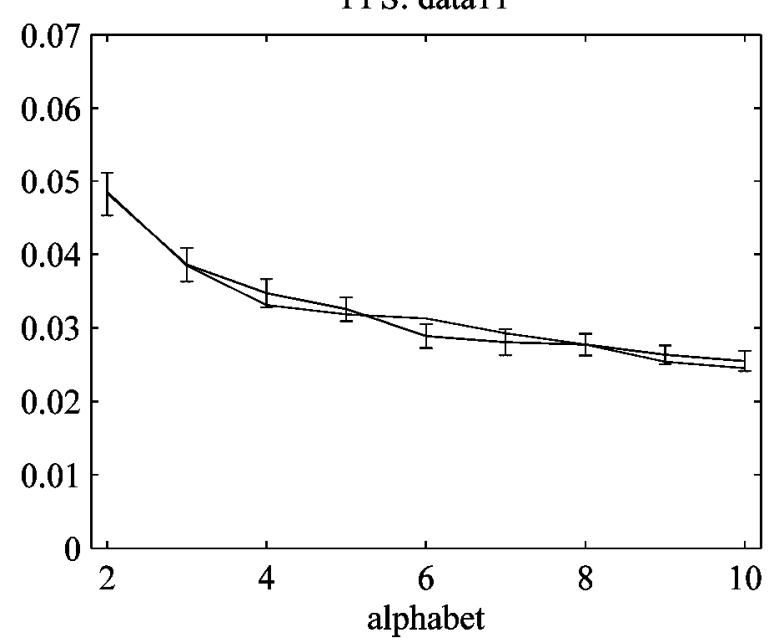

Fig. 11 


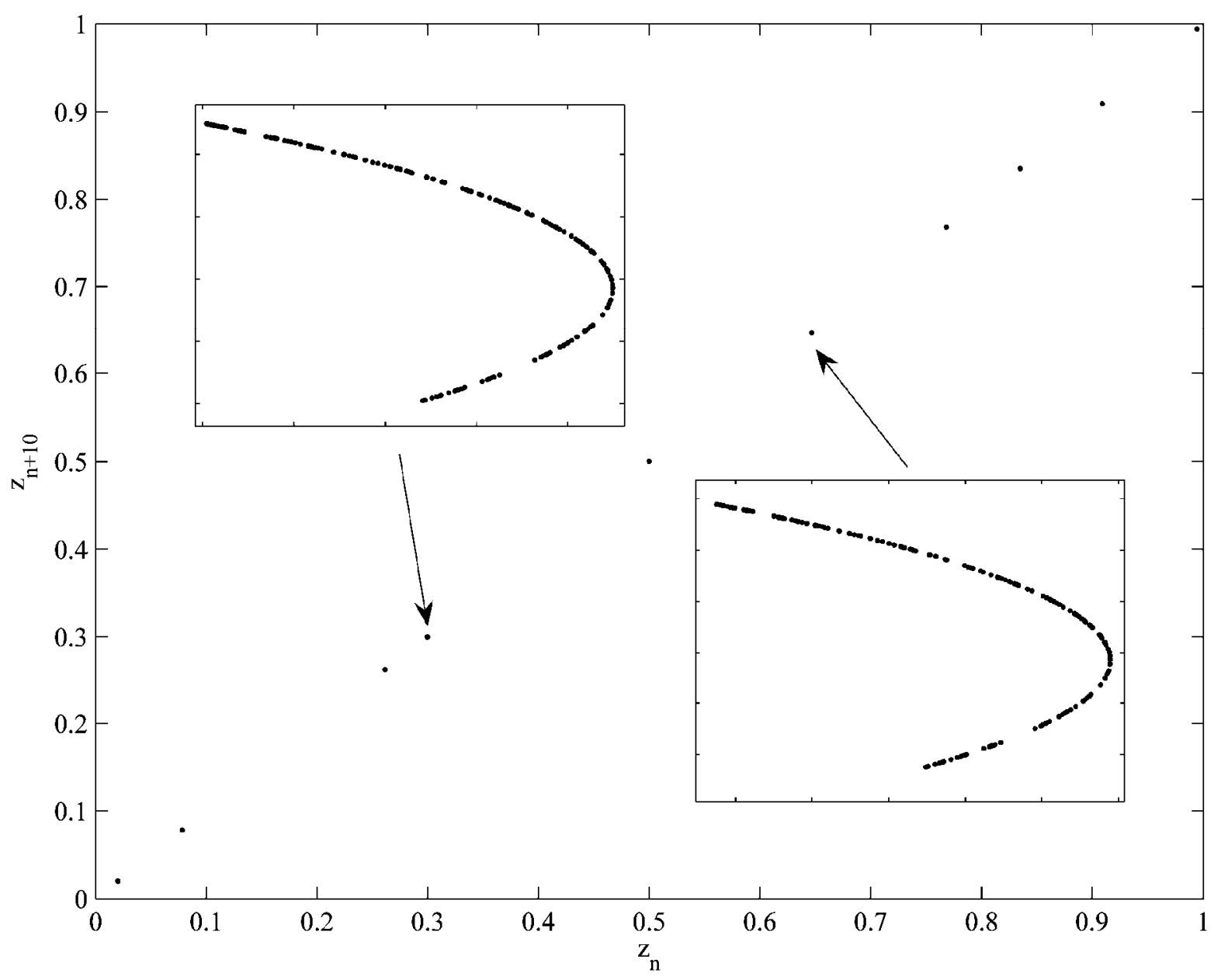

Fig. 12 